\title{
Selection of Proper Non-linear Kernel Parameter in Support Vector Machine Algorithm for Classifying the Internal Fault Type in Winding Power Transformer
}

\author{
J. Klomjit $^{\text {a }}$, S. Thongsuk ${ }^{\mathrm{b}, *}$, and A. Ngaopitakkul ${ }^{\mathrm{a}}$ \\ a Faculty of Engineering, King Mongkut's Institute of Technology Ladkrabang, Bangkok, 10520, Thailand \\ ${ }^{\mathrm{b}}$ Faculty of Industrial Technology, Rajabhat Rajanagarindra University, Chachoengsao, 24000, Thailand \\ *Corresponding Author: t surakit@yahoo.co.th
}

\begin{abstract}
This paper proposes the proper kernel function in a support vector machine (SVM), which is used to classify the internal fault type in power transformer. The Gaussian kernel and polynomial kernel that are two types of non-linear kernel parameter are compared in terms of the average accuracy and time of training process. The results are shown that the polynomial kernel parameter of SVM algorithm is able to classify the internal fault type with satisfactory accuracy, and it takes the average time less than the other. The benefit of using polynomial kernel can be applied for fault diagnosis in future.
\end{abstract}

Keywords: Power Transformer, Support vector machine, Kernel, Discrete Wavelet Transform, Fault.

\section{Introduction}

In recent year, artificial intelligent process has been applied for analyzing complex data. Many literatures have been reviewed for research proposed. Algorithm for fault diagnosis such as neural network ${ }^{(1-2)}$, fuzzy logic ${ }^{(3-4)}$, support vector machine ${ }^{(5-8)}$, and etc. has been used in many researches. These techniques are for achieving high accuracy of fault diagnosis. However, each technique has its own advantage and disadvantage on different types of system ${ }^{(9-11)}$. The feasibility study to apply fault diagnosis technique in practical system must be done to ensure satisfactory performance $^{(12)}$.

One of the algorithms that is widely used for fault classification is support vector machine algorithm. This algorithm can classify non-linear data by using kernel function capable of accommodating complicated data, so variety of data can be analyzed.

The purpose of this paper is to evaluate effect of kernel function on SVM by comparing two functions between Gaussian and polynomial kernel parameters, which are used to classify the internal fault type in power transformer. The fault conditions are simulated using ATP/EMTP program. The current waveforms obtained from the simulation are extracted using the Discrete Wavelet Transform (DWT). The DWT is usually employed to extract the high frequency component contained in the fault currents, and the DWT coefficients of the first scale that can detect fault are then investigated. The validity of the proposed algorithm is tested against various fault inception angles, fault locations, and faulty phases. The construction of the decision algorithm is detailed and implemented in various case studies that are based on Thailand's electricity transmission and distribution systems.

\section{Simulation on Power Transformer Winding}

For the simulation, the three-phase two-winding transformer 50 MVA, $115 / 23 \mathrm{kV}$ is employed in simulations with all parameters and configuration provided by a manufacturer. The BCTRAN model based on the test data can be obtained from the transformer manufacturers. The simulations are performed with various changes in system parameters as follows:

- The angles on phase A voltage waveform for the instant of fault inception are $30^{\circ}$.

- Internal fault types at the transformer windings (both primary and secondary) which are winding to ground fault and interturn fault are investigated. 
- The fault position designated on any phases of the transformer windings (both primary and secondary), is varied at the length of $10 \%, 30 \%, 50 \%, 70 \%$, and $90 \%$ measured from the line end of the windings.

- Fault resistance is $5 \Omega$.

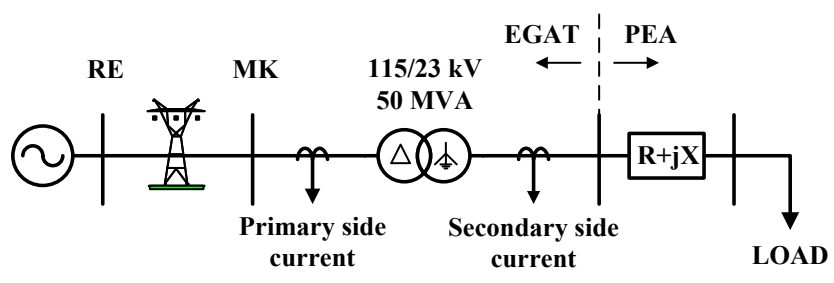

Fig. 1. Scheme under investigation.

\section{Decision algorithm}

The fault signals generated using ATP/EMTP are interfaced to MATLAB for the internal fault classification decision algorithm. The mother wavelet daubechies4 (db4) is employed to decompose the high frequency transient components, which are superimposed in the fault current signals, by DWT using the wavelet toolbox. After applying the DWT, coefficients obtained using DWT of signals are squared so that the abrupt change in the spectra can be clearly found. In previous paper ${ }^{(13)}$, this sudden change is used as an index for the occurrence of faults.

The internal fault classification decision algorithm is then constructed based on the support vector machine toolboxes in MATLAB. Before the decision algorithm process, support vector machine structure consists of 4 input as shown in Fig. 2. These inputs are the maximum coefficient of DWT at $1 / 4$ cycle of phase A, B, C and zero sequence provided to support vector machine model. The obtained values from DWT are normalized to between 0 and 1 for support vector machine process. For the next stage, we identify fault type and phase of internal fault in transformer by 6 models of support vector machine. Each model can identify different fault $\mathrm{s}$ as show in Table 1. Data mapping is an important part of support vector machine process as shown by flowchart in Fig. 3.

As previously mentioned, the objective of this paper is to evaluate effect of kernel function for the SVM. Non-linear kernel parameter in support vector machine commonly uses the Gaussian kernel or polynomial kernel. Finally, the decision algorithm can be verified with various case studies. There are 108 sets for winding to ground fault and 432 sets for interturn fault. The obtained results from decision algorithm that is employed to classify the internal fault are illustrated in Fig. 4.

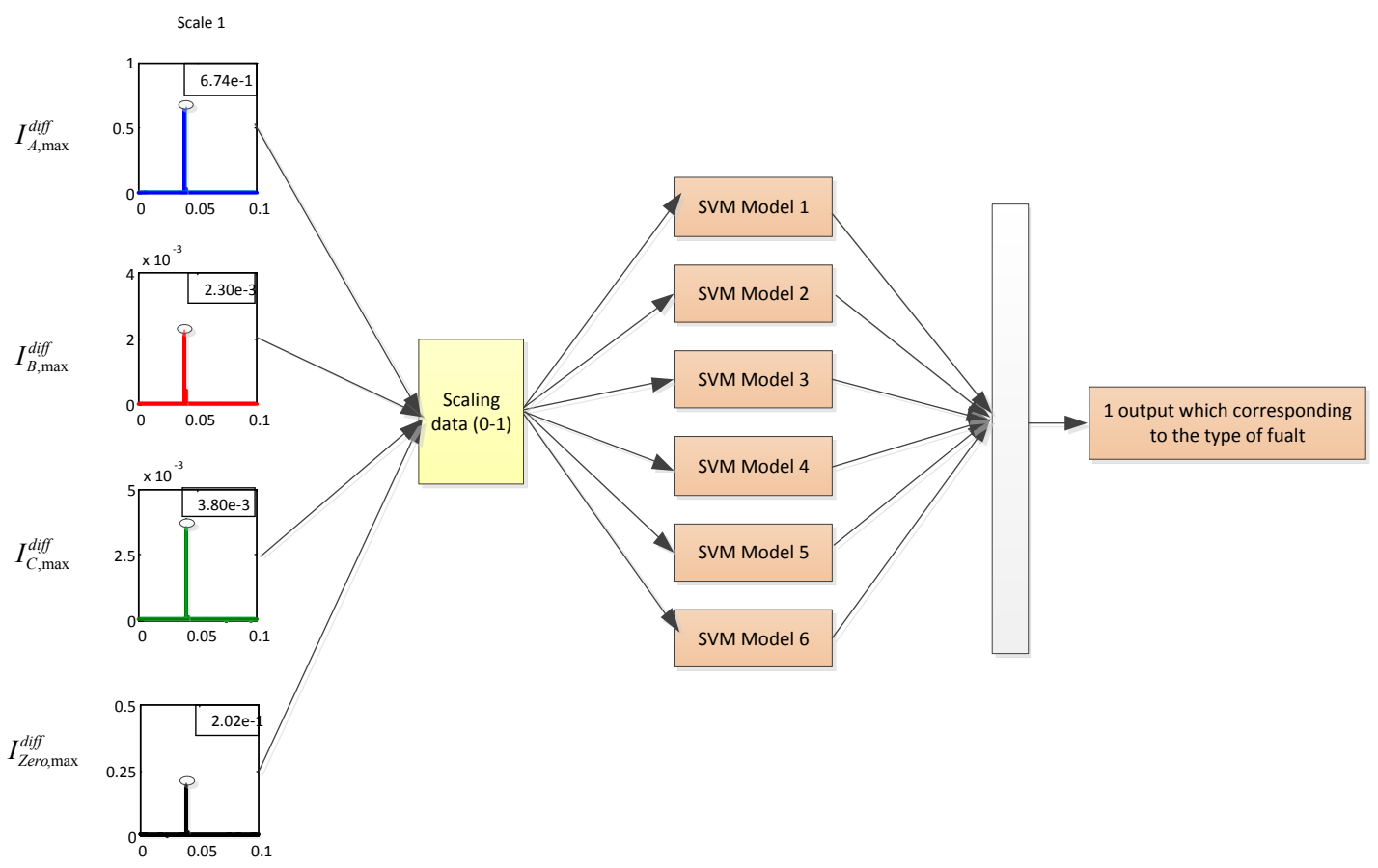

Fig. 2. Support vector machine structure for classifying the internal fault type. 
Table 1. Output model of support vector machine for fault type identifier.

\begin{tabular}{|c|c|c|c|}
\hline Models of SVM & Output of SVM & Classification of fault type & Types of fault \\
\hline \multirow{3}{*}{1} & 1 & Winding to ground fault at coil 1 (A-C) & WG-1 \\
\hline & 2 & Winding to ground fault at coil 4 (A) & WG-4 \\
\hline & 3 & Other fault & $\mathrm{N} / \mathrm{A}$ \\
\hline \multirow{3}{*}{2} & 4 & Winding to ground fault at coil 2 (B-A) & WG-2 \\
\hline & 5 & Winding to ground fault at coil 5 (B) & WG-5 \\
\hline & 6 & Other fault & N/A \\
\hline \multirow{3}{*}{3} & 7 & Winding to ground fault at coil 3 (C-B) & WG-3 \\
\hline & 8 & Winding to ground fault at coil $6(\mathrm{C})$ & WG-6 \\
\hline & 9 & Other fault & $\mathrm{N} / \mathrm{A}$ \\
\hline \multirow{3}{*}{4} & 10 & Interturn fault at coil 1 (A-C) & TT-1 \\
\hline & 11 & Interturn fault at coil $4(\mathrm{~A})$ & TT-4 \\
\hline & 12 & Other fault & $\mathrm{N} / \mathrm{A}$ \\
\hline \multirow{3}{*}{5} & 13 & Interturn fault at coil $2(\mathrm{~B}-\mathrm{A})$ & TT-2 \\
\hline & 14 & Interturn fault at coil $5(\mathrm{~B})$ & TT-5 \\
\hline & 15 & Other fault & $\mathrm{N} / \mathrm{A}$ \\
\hline \multirow{3}{*}{6} & 16 & Interturn fault at coil 3 (C-B) & TT-3 \\
\hline & 17 & Interturn fault at coil $6(\mathrm{C})$ & TT-6 \\
\hline & 18 & Other fault & N/A \\
\hline
\end{tabular}

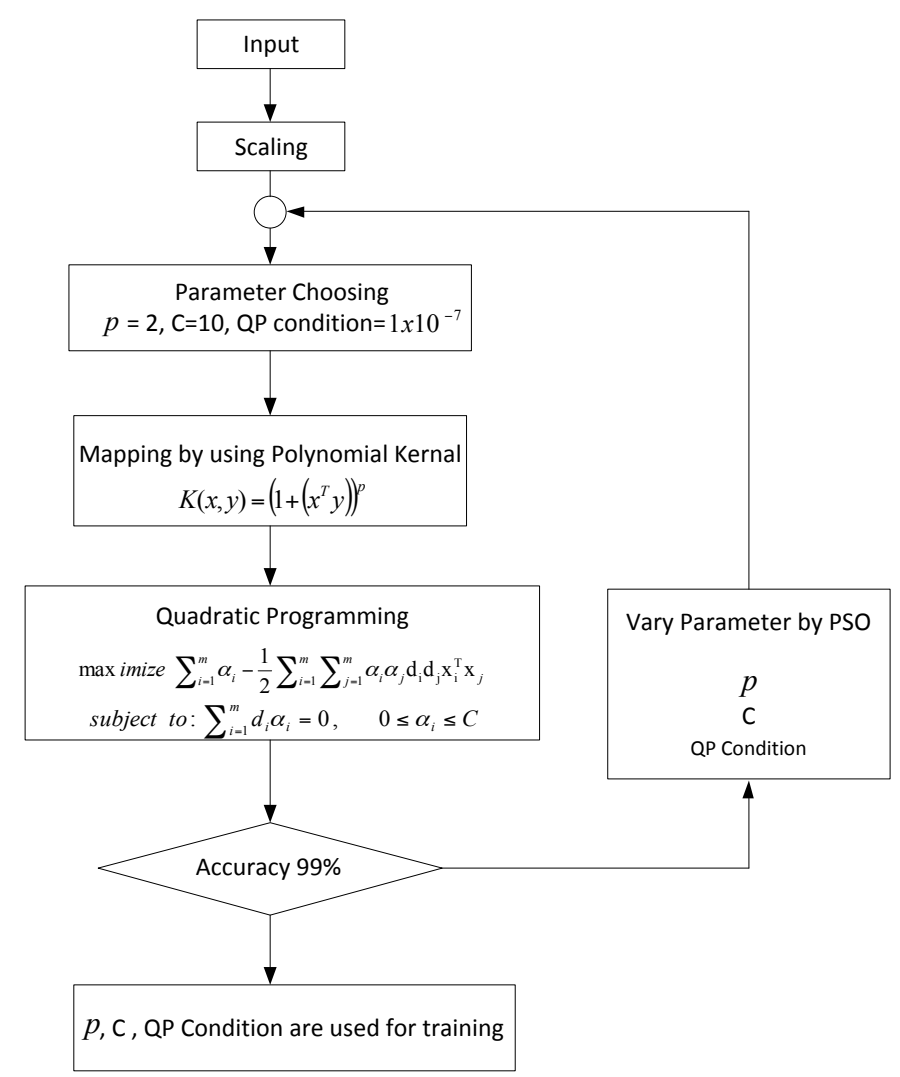

Fig. 3. Flow chart of support vector machine algorithm. 


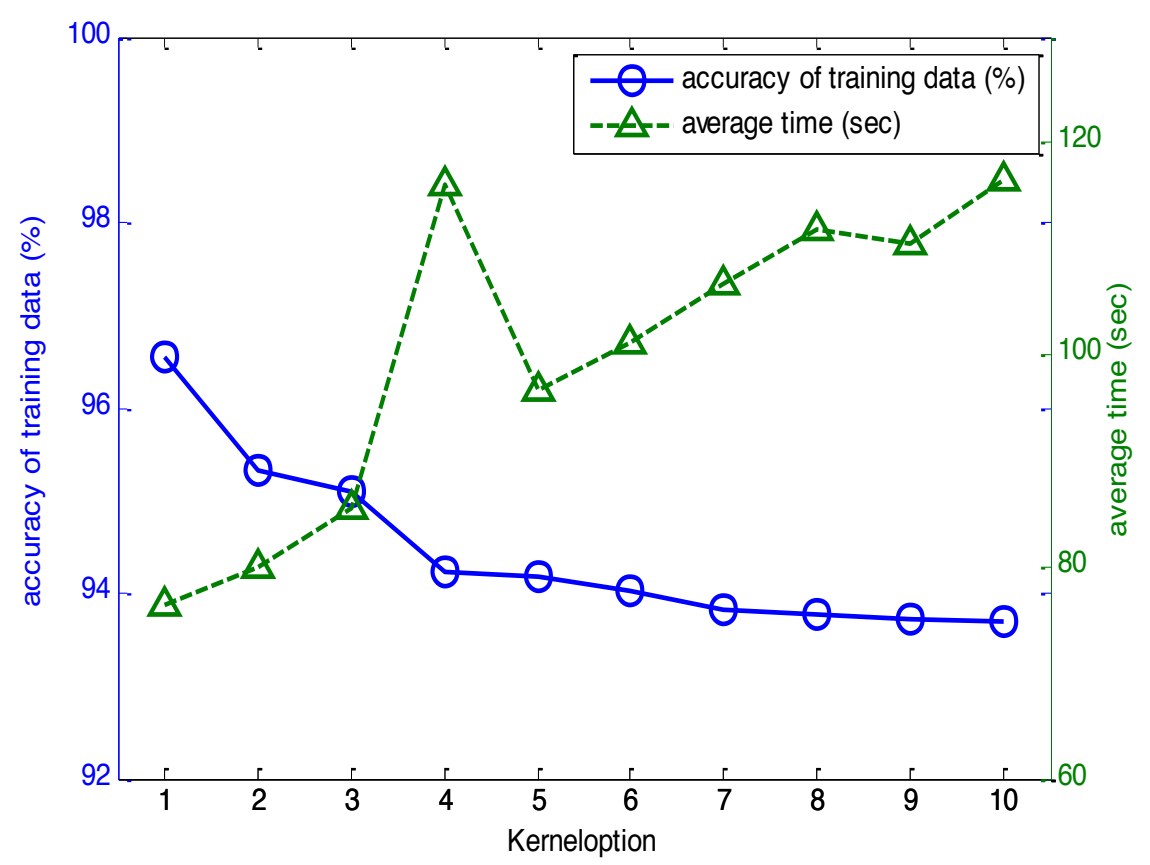

(a) Accuracy of Gaussian kernel

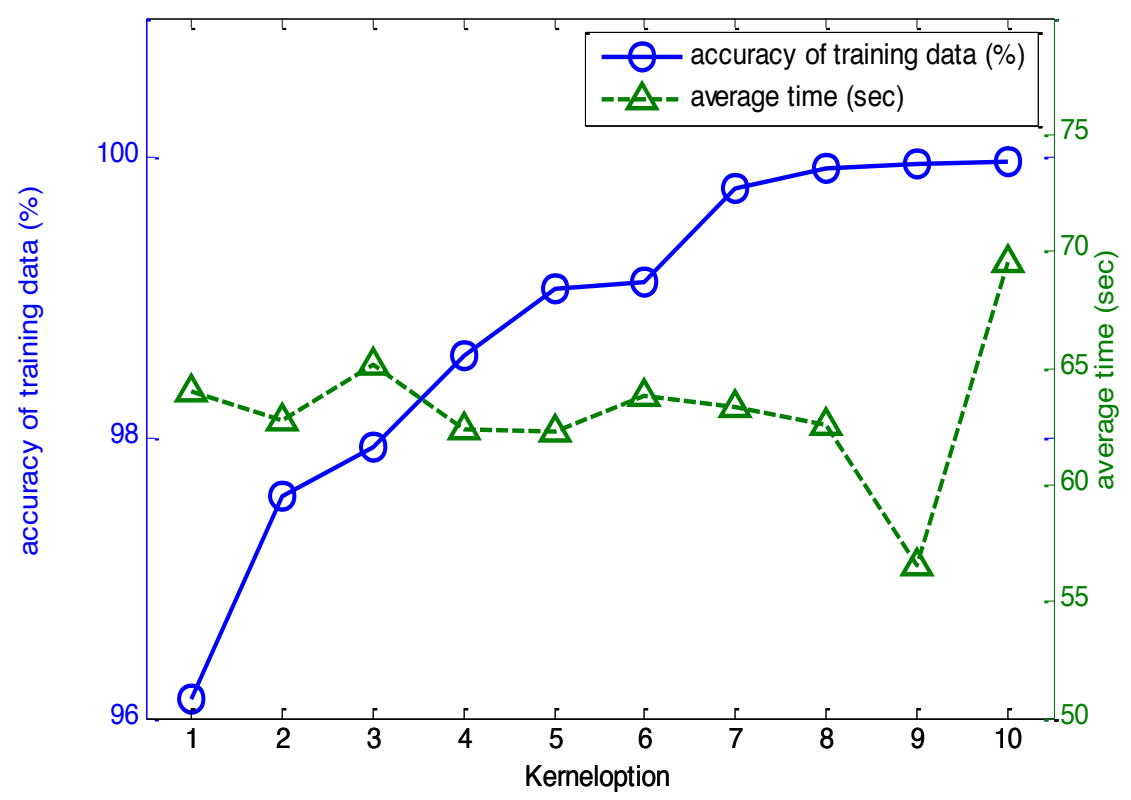

(a) Accuracy of polynomial kernel

Fig. 4. Relationship between kernel parameter and Accuracy of training data in fault identifier.

According to the Gaussian kernel parameter used in training process, relation between average accuracy of case studies and training time is shown in Fig. 4(a), it can be observed that, when the Gaussian kernel parameter increases, the average accuracy tends to decrease while the average training time tends to increase.
After the polynomial kernel parameter is used in training process, relation between average accuracy of case studies and training time can be also carried out and shown in Fig. 4(b), it can be observed that, when the polynomial kernel parameter increases, the average accuracy tends to increase while the average training time is slightly changed. 


\section{Conclusion}

One of parameter that has an effect on accuracy of SVM is kernel parameter. This paper investigated on effect of kernel parameter on performance of support vector machine algorithm by comparing Gaussian and polynomial kernel parameters. The algorithm is provided to set of training data to support vector machine model that can be compared in terms of time and accuracy. The obtained result reveals that polynomial kernel parameter is able to classify the internal fault type with high accuracy and requires less time for processing in comparison to Gaussian kernel parameter. From this result, support vector machine with polynomial kernel parameter can contribute satisfaction accuracy for fault diagnosis in power transformer.

\section{Acknowledgment}

The authors wish to gratefully acknowledge financial support for this research from the King Mongkut's Institute of Technology Ladkrabang Research fund, Thailand. This work is also partially supported by Rajabhat Rajanagarindra University Research fund.

\section{References}

(1) Zhenyuan Wang, Yilu Liu, and P.J. Griffin : "Neural net and expert system diagnose transformer faults", IEEE Computer Applications in Power, Vol. 13, No. 1, pp. 50-55, 2000.

(2) Ke Meng, Zhao-Yang Dong, Dian-Hui Wang, and Kit-Po Wong : “A Self-Adaptive RBF Neural Network Classifier for Transformer Fault Analysis", IEEE Transactions on Power Systems, Vol. 25, No. 3, pp. 1350-1360, 2010.

(3) R. Naresh, V. Sharma, and M. Vashisth : "An Integrated Neural Fuzzy Approach for Fault Diagnosis of Transformers", IEEE Transactions on Power Delivery, Vol. 23, No. 4, pp. 2017-2024, 2008.

(4) R.A. Hooshmand, M. Parastegari, and Z. Forghani : "Adaptive neuro-fuzzy inference system approach for simultaneous diagnosis of the type and location of faults in power transformers", IEEE Electrical Insulation Magazine, Vol. 28, No. 5, pp.32-42, 2012.

(5) A.M. Shah, and B.R. Bhalja : "Discrimination Between Internal Faults and Other Disturbances in Transformer Using the Support Vector Machine-Based Protection
Scheme”, IEEE Transactions on Power Delivery, Vol. 28, No. 3, pp. 1508-1515, 2013.

(6) H.B. Zheng, R.J. Liao, S. Grzybowski, and L.J. Yang : Fault diagnosis of power transformers using multi-class least square support vector machines classifiers with particle swarm optimization", IET Electric Power Applications, Vol. 5, No. 9, pp. 691-696, 2011.

(7) C. Koley, P. Purkait, and S. Chakravorti : "Wavelet-aided SVM tool for impulse fault identification in transformers", IEEE Transactions on Power Delivery, Vol. 21, No. 3, pp. 1283-1290, 2006.

(8) M. Bigdeli, M. Vakilian, and E. Rahimpour : "Transformer winding faults classification based on transfer function analysis by support vector machine", IET Electric Power Applications, Vol.6, No.5, pp. 268-276, 2012.

(9) N.G. Chothani, B.R. Bhalja, and U.B. Parikh : "New fault zone identification scheme for busbar using support vector machine", IET Generation, Transmission \& Distribution, Vol. 5, No. 10, pp. 1073-1079, 2011.

(10)D. Thukaram, H.P. Khincha, and H.P. Vijaynarasimha : "Artificial neural network and support vector Machine approach for locating faults in radial distribution systems", IEEE Transactions on Power Delivery, Vol. 20, No. 2, pp. 710-721, 2005.

(11)F.R. Gomez, A.D. Rajapakse, U.D. Annakkage, and I.T. Fernando : "Support Vector Machine-Based Algorithm for Post-Fault Transient Stability Status Prediction Using Synchronized Measurements", IEEE Transactions on Power Systems, Vol. 26, No. 3, pp. 1474-1483, 2011.

(12) R.A. Ghunem, R. El-Shatshat, and O. Ozgonenel : "A Novel Selection Algorithm of a Wavelet-Based Transformer Differential Current Features", IEEE Transactions on Power Delivery, Vol. 29, No. 3, pp. 1120-1126, 2014.

(13)A. Ngaopitakkul and A. Kunakorn : "Internal Fault Classification in Transformer Windings using Combination of Discrete Wavelet Transforms and Back-propagation Neural Networks", International Journal of Control, Automation, and Systems (IJCAS), Vol. 4, No. 2, pp. 365-371, 2006. 